\title{
Nod1, but not the ASC inflammasome, contributes to induction of IL-1 $\beta$ secretion in human trophoblasts after sensing of Chlamydia trachomatis
}

\author{
PB Kavathas ${ }^{1,2,4}$, CM Boeras ${ }^{1,4}$, MJ Mulla ${ }^{3}$ and VM Abrahams ${ }^{3}$
}

\begin{abstract}
Chlamydia trachomatis (Ct) is an obligate intracellular bacterial pathogen. Previously, we showed that infection of human trophoblast cells by $\mathrm{Ct}$ triggers the secretion of the pro-inflammatory cytokine, interleukin (IL)-1 $\beta$. The aim of this study was to understand the innate immune pathways involved in trophoblast production of IL-1 $\beta$ after Ct infection. The approach we took was to inhibit the expression or function of the key Toll-like receptors (TLRs), Nod-like receptors, and inflammasome components that have been associated with chlamydia infection. In this study, we report that Ct-induced trophoblast IL-1 $\beta$ secretion is associated with the transcription of IL-1 $\beta$ mRNA, the translation and processing of pro-IL-1 $\beta$, and the activation of caspase-1. In addition, we demonstrate that Ct-induced IL-1 $\beta$ production and secretion by the trophoblast is independent of TLR2, TLR4, MyD88, and the Nalp3/ASC inflammasome. Instead we report, for the first time, the importance of Nod1 for mediating trophoblast IL-1 $\beta$ secretion in response to a Ct infection.
\end{abstract}

\section{INTRODUCTION}

Chlamydia trachomatis $(\mathrm{Ct})$ is an obligate intracellular Gramnegative bacterium that is the leading cause of bacterial sexually transmitted infections. ${ }^{1}$ Because it is largely asymptomatic, many individuals without access to routine healthcare go untreated. If untreated, $\mathrm{Ct}$ infection can lead to pelvic inflammatory disease, sterility, and ectopic pregnancy. ${ }^{2}$ Moreover, chlamydia is increasingly becoming seen as an emerging pathogen during pregnancy because of its association with adverse pregnancy outcomes and vertical transmission. ${ }^{3,4}$ Although Chlamydia mainly infects epithelial mucosa, it can also infect other cell types. Indeed, clinical studies have shown that $\mathrm{Ct}$, as well as other strains like Chlamydia pneumoniae, can be detected in placental tissue, ${ }^{5-9}$ and experimental studies have demonstrated that $\mathrm{Ct}$ can infect human placental trophoblast cells. ${ }^{10,11}$ Thus, characterizing the innate immune response to $\mathrm{Ct}$ infection is important for understanding host-pathogen interactions. Furthermore, understanding the mechanisms by which $\mathrm{Ct}$ impacts trophoblast function may have clinical significance by helping us to better appreciate the progression of a Ct infection during pregnancy.

Microbes are initially sensed by cells of the innate immune system through binding to pattern recognition receptors (PRRs), such as the Toll-like receptors (TLRs) and Nod-like receptors (NLRs), resulting in a range of responses that include the secretion of pro-inflammatory cytokines and chemokines, the production of anti-microbial factors, and the induction of cell death by apoptosis, autophagy, or pyroptosis. ${ }^{12-14}$ However, non-immune cells, such as the trophoblast, also express TLRs and NLRs, and through these, can mount innate immune responses towards pathogens and infectious components. ${ }^{15,16}$ The TLRs are transmembranal PRRs, which allow for the extracellular and endosomal recognition of microbes or infectious components, and all but TLR3 signal through the adapter protein, myeloid differentiation primary response gene 88 (MyD88). ${ }^{17}$ The cytoplasmic-based NLRs function as intracellular receptors that respond to microbes or microbial components, which have gained access to the cell's cytoplasm. ${ }^{18}$

\footnotetext{
${ }^{1}$ Department of Laboratory Medicine, Yale University School of Medicine, New Haven, Connecticut, USA. ${ }^{2}$ Department of Immunobiology, Yale University School of Medicine, New Haven, Connecticut, USA. ${ }^{3}$ Department of Obstetrics, Gynecology, and Reproductive Sciences, Yale University School of Medicine, New Haven, Connecticut, USA. ${ }^{4}$ These authors contributed equally to this work. Correspondence: PB Kavathas (paula.kavathas@yale.edu) or VM Abrahams (vikki.abrahams@yale.edu) 
The most well-characterized NLRs in terms of microbial sensing are the Nod proteins, Nod1 and Nod2, and Nalp3 (Nlrp3). Nod 1 and Nod2 induce inflammatory responses through the common adapter protein, Rip $2 .{ }^{18} \mathrm{Nalp} 3$ activation leads to the formation of the inflammasome, a protein platform that also contains the adapter protein, ASC (apoptosis-associated speck-like protein containing a CARD) and the non-apoptotic, caspase-1. Activation of the inflammasome specifically mediates the processing of intracellular pro-interleukin (IL)-1 $\beta$ (and pro-IL-18) into its active, secreted form. ${ }^{19}$

Several TLRs and NLRs have been reported to sense Ct and the related murine strain, Chlamydia muridarum. TLR2, which is normally located on the plasma membrane, and MyD88, are specifically recruited to the inclusion membrane during a productive infection and are required for Ct-induced IL-8 secretion..$^{20}$ Other studies have also implicated TLR2, TLR4, and MyD88 in chlamydia-mediated inflammation and pathology. ${ }^{21-26}$ In addition to the TLR/MyD88 pathway, intracellular Nod1 has been shown to have a role in Ct-induced inflammation, ${ }^{27,28}$ while Nod1 and Nod2 can sense C. pneumoniae. ${ }^{29,30}$ Some studies report that Nalp3 is important for Ct-induced caspase- 1 activation and IL- $1 \beta$ production. ${ }^{29,31}$

We recently demonstrated that $\mathrm{Ct}$ infection of human trophoblast cells leads to the induction of IL- $1 \beta$ secretion, ${ }^{11}$ which is consistent with studies in other cell types. ${ }^{23,29,32,33}$ The trophoblast is also known to produce IL- $1 \beta$ via the inflammasome component, ASC in response to the Nalp3 agonist, monosodium urate (MSU). ${ }^{34}$ Therefore, the aim of this study was to understand the molecular basis for the secretion of the pro-inflammatory cytokine IL- $1 \beta$ after Ct infection of human trophoblast cells. The approach we took was to inhibit the expression or function of the key TLRs and NLRs that have been associated with $\mathrm{Ct}$ infection, ${ }^{20-31}$ and to determine the effect on IL- $1 \beta$ production. In this study, we report that Ct-induced IL-1 $\beta$ production in the trophoblast is independent of TLR2, TLR4, MyD88, Nalp3, and ASC. However, activation of Nod1 by Ct mediates IL- $1 \beta$ secretion by human trophoblast cells.

\section{RESULTS}

Ct infection of trophoblast cells induces IL-1 $\beta$ expression, processing, and secretion

For IL-1 $\beta$ to be secreted, it must first be expressed as a precursor pro-IL-1 $\beta$, which is then cleaved into its bioactive form; and typically IL- $1 \beta$ processing is mediated by active caspase- $1 .{ }^{19}$ We, therefore, examined whether our previous observations of Ct-mediated IL- $1 \beta$ secretion by the trophoblast ${ }^{11}$ was associated with its induction and processing. For this, we used two human first trimester trophoblast cell lines, HTR8 and Sw.71, which we had previously shown to be readily infected by Ct serovar D. ${ }^{11}$ For this current study, the trophoblast cells lines were either not infected, or infected with a rifampin resistant strain of $\mathrm{Ct}$ serovar D at a multiplicity of infection of 1 , and after $24 \mathrm{~h}$, as we have previously published, ${ }^{11} \sim 60 \%$ of the cells were infected (data not shown).

We found that after Ct infection, both mRNA, pro-IL-1 $\beta$, and processed IL- $1 \beta$ were induced. After $24 \mathrm{~h}$, Ct infection of the HTR8 trophoblast cells significantly induced IL- $1 \beta$ mRNA expression (Figure 1a). Ct infection of the HTR8 and Sw.71 cells also significantly induced IL- $1 \beta$ secretion into the culture supernatants after 48 and $36 \mathrm{~h}$, respectively (Figure $\mathbf{1 b}, \mathbf{c}$ ). In addition to secreting IL- $1 \beta$ more rapidly, the Sw.71 cells secreted much higher levels of IL-1 $\beta$ than the HTR8 cells (Figure $\mathbf{1 b}, \mathbf{c}) .{ }^{11}$ Next, we examined the levels of intracellular pro-IL-1 $\beta(31 \mathrm{kDa})$, active-IL-1 $\beta(17 \mathrm{kDa})$, and active-caspase- $1(20 \mathrm{kDa})$ protein by western blot analysis and densitometry. Uninfected HTR8 and Sw.71 trophoblast cells expressed no or minimal levels of pro-IL-1 $\beta$; however, after Ct infection as early as $12 \mathrm{~h}$, there was an induction of pro-IL- $1 \beta$ expression, and this continued to increase at 24 and $48 \mathrm{~h}$ post infection (Figure 1d,e). Both the HTR8 and Sw.71 cells had detectable amounts of intracellular active IL-1 $\beta$ and active caspase-1, regardless of infection status, and levels were not significantly different between infected and uninfected cells (Figure 1d,e). Therefore, we examined IL-1 $\beta$ and caspase- 1 protein expression in the culture supernatants by western blot. Interestingly, in the uninfected Sw.71 cell supernatants, there were detectable levels of pro-IL-1 $\beta$, but little, if any, active IL-1 $\beta$ or active caspase-1 (Figure 1f), unlike in the cell lysates (Figure 1e). In the Sw.71 cell supernatants $36 \mathrm{~h}$ post-infection, however, there were significantly higher levels of pro-IL-1 $\beta$, active-IL-1 $\beta$, and active caspase- 1 compared with the non-infected cells (Figure 1f).

\section{Ct-induced trophoblast IL-1 $\beta$ expression and secretion is not dependent upon TLR2, TLR4, or MyD88}

Having established that $\mathrm{Ct}$ infection of trophoblast cells led to the induction of IL-1 $\beta$ expression, processing, and secretion, we sought to determine which innate immune receptors activated by Ct were contributing to this response. PRRs that have been reported to be activated by chlamydia are TLR2, TLR4, Nod1, Nod2, and the Nalp3 inflammasome. ${ }^{20-31}$ We first tested whether TLR2 and TLR4 were involved using trophoblast cell lines stably transfected to express a TLR2-dominant negative (DN) and TLR4-DN that we had previously created and characterized in the trophoblast cell line 3A and HTR8, respectively. ${ }^{35-37}$ Using these cell lines, we previously showed that TLR2 is involved in trophoblast cell apoptosis induced by Gram-positive bacterial peptidoglycan, ${ }^{37}$ because the response was blocked in the TLR2-DN cells. TLR4 mediates trophoblast anti-phospholipid antibody-induced inflammation, ${ }^{36}$ because the response was blocked in the TLR4-DN cells. There was no significant difference in the levels of Ct-induced intracellular pro-IL-1 $\beta$ in the TLR2-DN cells (Figure 2a) or TLR4-DN cells (Figure 2b) when compared with the wild-type cells. Similarly, there was no significant difference in the secreted levels of Ct-induced IL-1 $\beta$ or IL-8 in the TLR2-DN cells (Figure 2c,e) or TLR4-DN cells (Figure 2d,f) when compared with the wild-type cells.

As neither TLR2 nor TLR4 appeared to have a role in Ct-induced IL- $1 \beta$ production, we tested the potential role of other TLRs by inhibiting the function of the common adapter protein, MyD88. ${ }^{17}$ For this, we established a HTR8 trophoblast cell line, stably transfected to express a MyD88-DN, which we have previously characterized. ${ }^{35,36}$ The ability of 

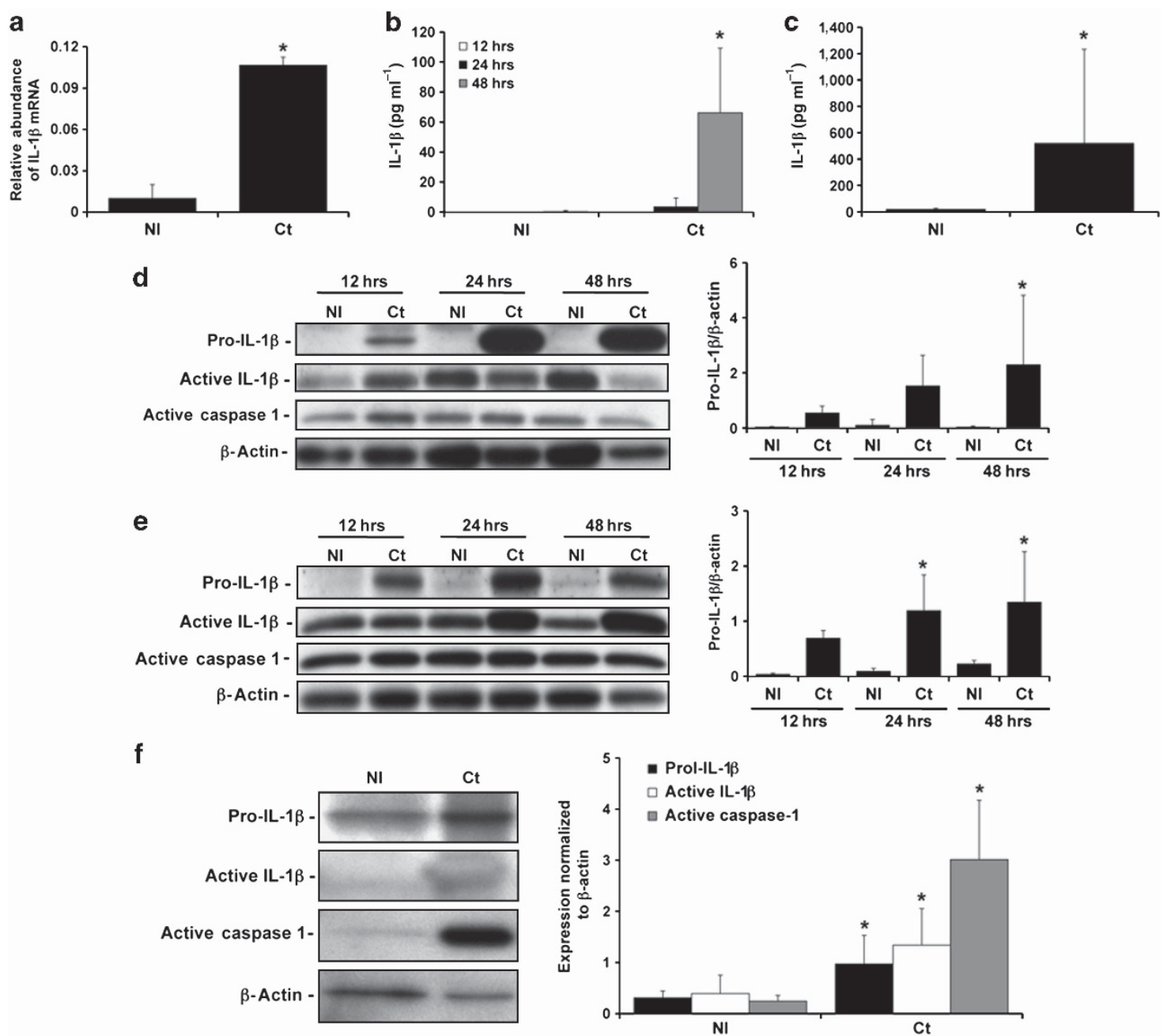

Figure 1 Chlamydia infection of human trophoblast cell lines leads to induction of interleukin (IL)-1 $\beta$ expression and IL-1 $\beta$ processing. Trophoblast cells were either non-infected (NI) or infected with Chlamydia trachomatis $(\mathrm{Ct})$, after which supernatants were collected and either RNA or protein extracted from cells. (a) Ct infection of HTR8 cells significantly induced IL-1 $\beta$ mRNA expression after $24 \mathrm{~h}$ as determined by quantitative real-time reverse-transcriptase-PCR; and (b) significantly induced IL-1 $\beta$ secretion after $48 \mathrm{~h}$ as determined by enzyme-linked immunosorbent assay. (c) Ct infection of Sw.71 cells significantly induced IL-1 $\beta$ secretion after $36 \mathrm{~h}$. $\left(n=3 ;{ }^{*} P<0.05\right.$ relative to the NI control). Cell lysates from (d) HTR8 and (e) Sw.71 cells after either NI or infection with Ct were evaluated for pro-IL-1 $\beta(31 \mathrm{kDa})$, active IL-1 $\beta(17 \mathrm{kDa})$ and active caspase-1 (20 kDa) expression by western blot (representative blots are shown). Bar charts show quantification of protein expression as determined by densitometry and normalized to $\beta$-actin $\left(n=3\right.$; ${ }^{\star} P<0.05$ relative to the $\mathrm{NI}$ control). (f) Cell supernatants from Sw.71 cells either NI or infected with Ct for $36 \mathrm{~h}$ were evaluated for pro- and active IL-1 $\beta$ and active caspase-1 expression by western blot. Bar charts show quantification of protein expression as determined by densitometry and normalized to $\beta$-actin $\left(n=4 ;{ }^{*} P<0.05\right.$ relative to the $\mathrm{NI}$ control).

Ct infection to induce trophoblast IL-1 $\beta$ RNA expression (Figure 3a) and intracellular pro-IL-1 $\beta$ protein expression (Figure 3b) was unaffected by the presence of the MyD88-DN. Similarly, the levels of Ct-induced IL- $1 \beta$ secretion were the same in the wild-type and MyD88-DN trophoblast cells (Figure 3c). However, the presence of the MyD88-DN significantly reduced the ability of Ct infection to induce trophoblast IL-8 secretion (Figure 3d).

\section{Ct-induced trophoblast IL-1 $\beta$ secretion is not dependent upon ASC or Nalp3}

Having established that the TLR/MyD88 pathway was not involved in Ct-induced IL-1 $\beta$ production, we investigated the potential role of the Nalp3 inflammasome. We have previously reported that the Nalp3 agonist, MSU, can induce IL- $1 \beta$ production via ASC in the Sw.71 trophoblast cell line, suggesting that the Nalp3 inflammasome is functional in these cells. ${ }^{34}$ Thus, for this current study, we used the same stable trophoblast cells expressing either small hairpin RNA (shRNA) specific for ASC (sh-ASC) or a non-targeting control (sh-control), and by western blot-found good knockdown of the ASC protein (Figure 4a). Nonetheless, there was no significant difference in the levels of secreted IL-1 $\beta$ in the sh-ASC cells when compared with the sh-control cells after Ct infection (Figure $4 \mathbf{b}$ ). In addition, the HTR 8 cells, which secrete IL- $1 \beta$ in response to Ct infection (Figure 1b), ${ }^{11}$ lack ASC protein. ${ }^{34}$ Stable cell lines expressing either shRNA specific for Nalp3 (sh-Nalp3) or a non-targeting control (sh-mut) were also established. 
a

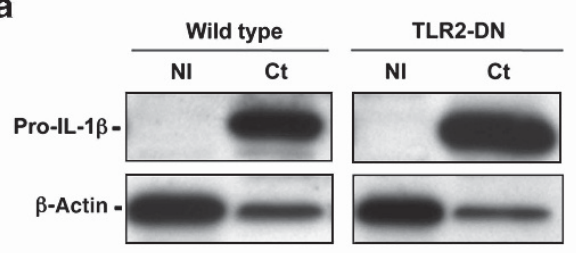

b
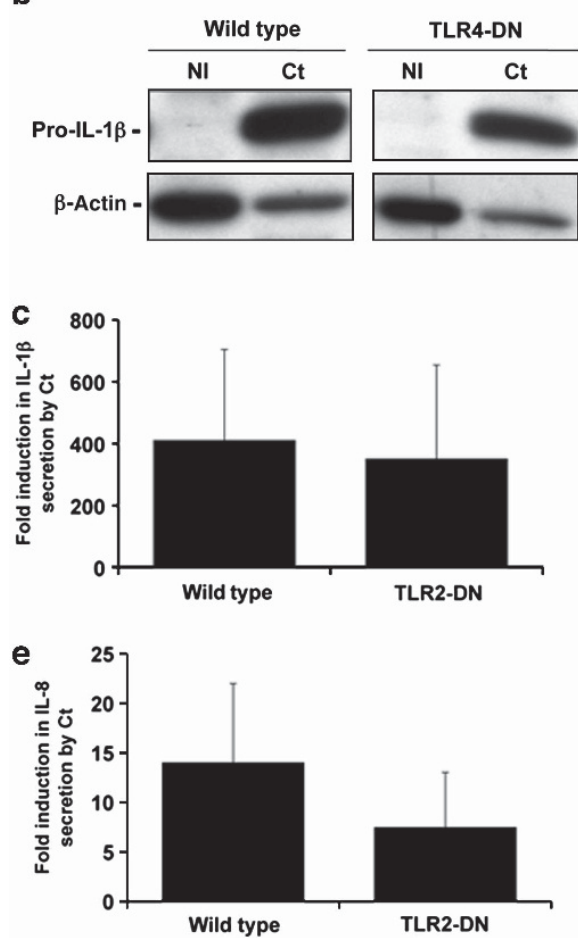
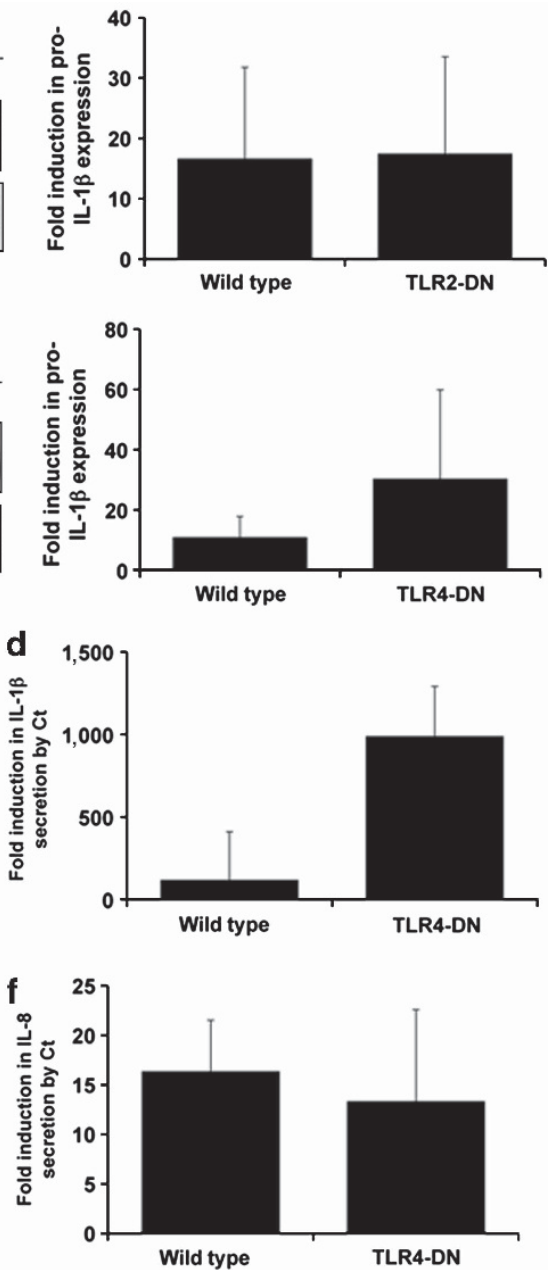

Figure 2 Chlamydia-induced trophoblast pro-interleukin (IL)-1 $\beta$ expression and secretion of IL-1 $\beta$ and IL-8 is independent of Toll-like receptor 2 (TLR2) and TLR4. Wild-type trophoblast (3A and HTR8) and trophoblast cells transfected to express either a TLR2-dominant negative (DN; in 3A cells) or a TLR4-DN (in HTR8 cells) were either non-infected (NI) or infected with Chlamydia trachomatis (Ct) for $48 \mathrm{~h}$. (a and b) Cell lysates were evaluated for pro-IL-1 $\beta$ expression by western blot (representative blots are shown). Bar charts show quantification of pro-IL-1 $\beta$ levels, as determined by densitometry and normalized to $\beta$-actin. Culture supernatants were analyzed for (c and d) IL-1 $\beta$ and (e and f) IL-8 levels by enzyme-linked immunosorbent assay. Data are pooled from three independent experiments and no significance was observed between the responses of wild type and TLR2-DN or TLR4-DN cells to Ct infection.

We were unable to determine Nalp3 protein knockdown as, to our knowledge, there are no reliable commercially available antibodies to Nalp3. However, Nalp3 mRNA levels were significantly lower in the sh-Nalp 3 cells when compared with the sh-mut cells (Figure 4c). Although MSU-induced IL- $1 \beta$ secretion was significantly reduced in the sh-Nalp3 cells when compared with the sh-mut cells (Figure 4d), we could not detect an effect on Ct-induced IL- $1 \beta$ secretion (Figure $4 \mathbf{e}$ ) in the cells containing Nalp3 shRNA.

\section{IL-1 $\beta$ secretion after trophoblast Ct infection is mediated by Nod1}

Both the Sw.71 and HTR8 trophoblast cell lines express Nod1, whereas the Sw.71 cells, but not the HTR8 cells, express Nod2. ${ }^{38}$ Having found that neither Nalp3 nor ASC were involved in the Ct-induced IL-1 $\beta$ response, we, therefore, turned our attention to determining whether $\mathrm{Ct}$ stimulation of Nod1 could be inducing
IL-1 $\beta$ secretion. Thus, a stable Sw.71 cell line expressing shRNA directed against Nod1 was established (sh-Nod1). As shown in Figure 5a, there was good reduction of Nod1 protein expression in the sh-Nod1 cells when compared with the sh-control cells. At $36 \mathrm{~h}$ post-infection, secretion of IL- $1 \beta$ (Figure $5 \mathbf{b}$ ) and IL-8 (Figure 5c) was significantly reduced in the sh-Nod1 cells when compared with the sh-control. By contrast, Ct-induced G-CSF (granulocyte colony-stimulating factor) secretion was unaffected by Nod1 knockdown (Figure 5d). As shown in Figure 5e, analysis of the supernatants by western blot revealed that Nod1 knockdown significantly reduced the secreted levels of active IL- $1 \beta$ induced by $\mathrm{Ct}$ infection. However, there was no significant difference in the $\mathrm{Ct}$-induction of secreted pro-IL-1 $\beta$ or active caspase- 1 in the sh-Nod 1 cells when compared with the sh-control cells. Therefore, signaling through the Nod1 receptor after $\mathrm{Ct}$ infection of human trophoblast cells is important for IL- $1 \beta$ secretion. 


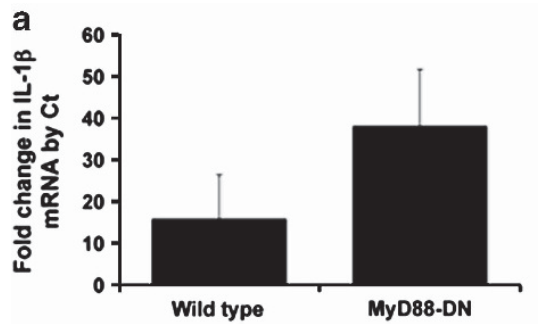

C

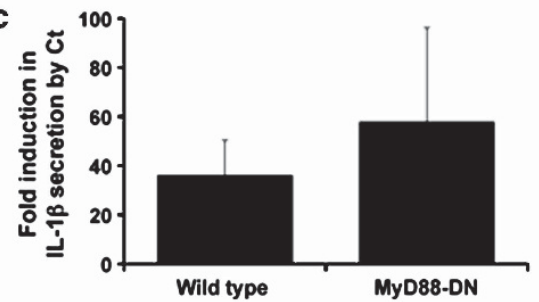

b
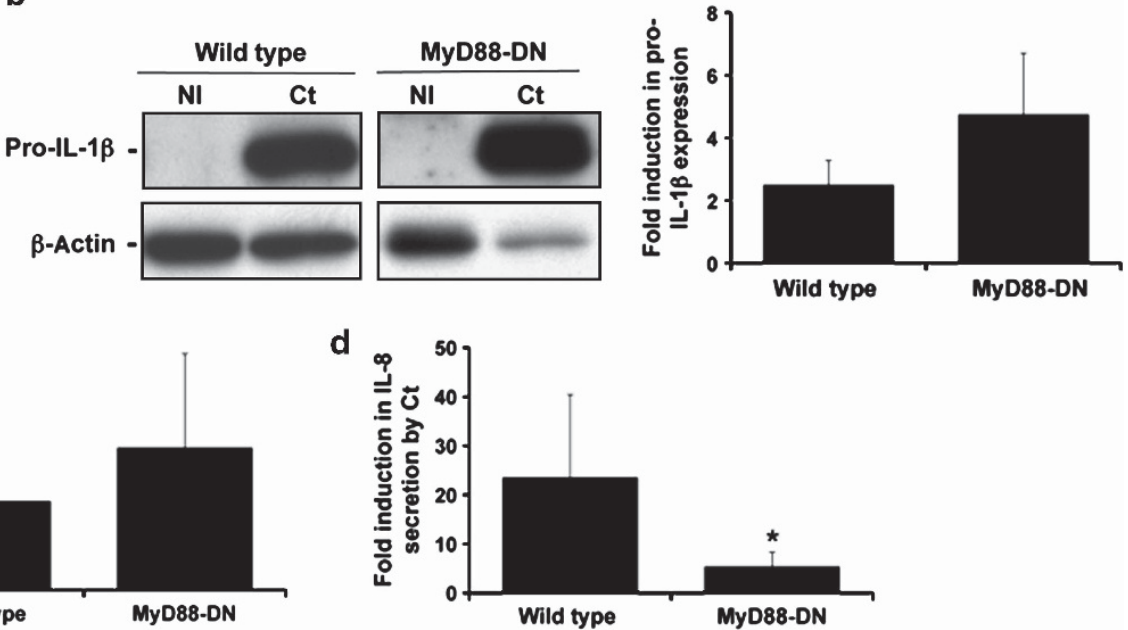

Figure 3 Chlamydia-induced trophoblast interleukin (IL)-8 secretion, but not IL-1 $\beta$ expression or secretion, is dependent on myeloid differentiation primary response gene 88 (MyD88). Wild-type HTR8 trophoblast and HTR8 cells transfected to express a MyD88-dominant negative (DN) were either non-infected (NI) or infected with Chlamydia trachomatis (Ct) for $48 \mathrm{~h}$. (a) RNA was analyzed for IL-1 $\beta$ mRNA levels by quantitative real-time reverse-transcriptase-PCR; (b) cell lysates were analyzed for pro-IL-1 $\beta$ expression levels by western blot (representative blots are shown) and densitometry; and culture supernatants were measured for (c) IL-1 $\beta$ and (d) IL-8 by enzyme-linked immunosorbent assay. Data are pooled from three independent experiments; ${ }^{\star} P<0.05$ relative to the wild-type cells.

a

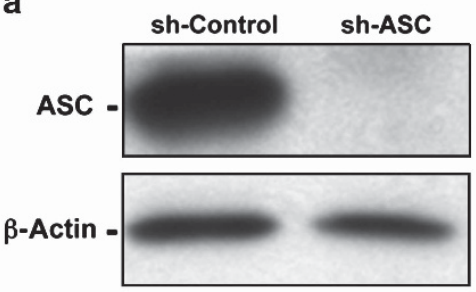

C

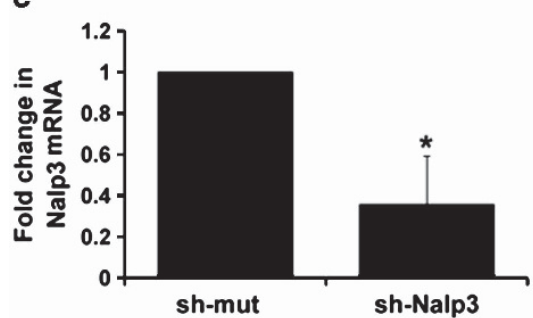

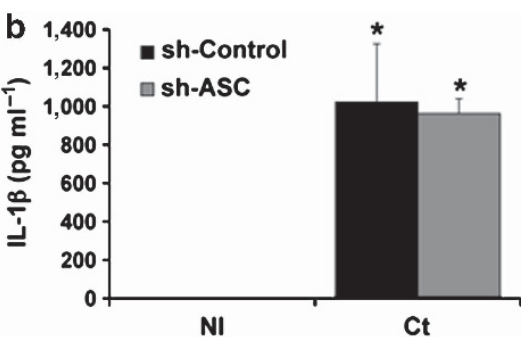

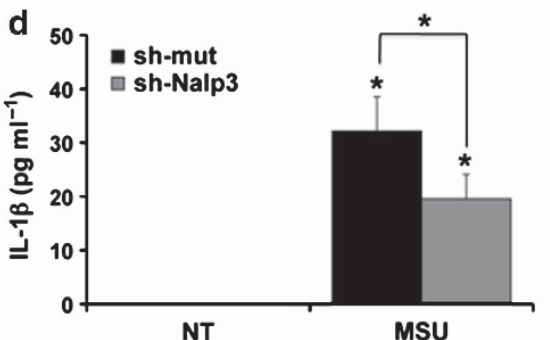

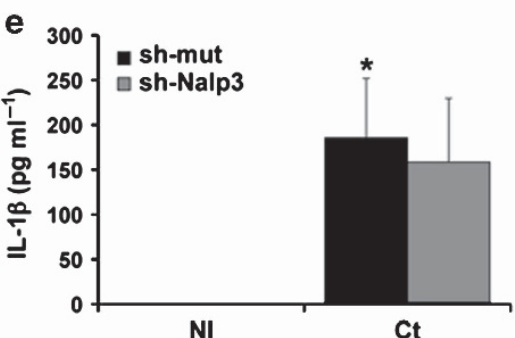

Figure 4 Chlamydia-induced trophoblast interleukin (IL)-1 $\beta$ secretion is not dependent on ASC (apoptosis-associated speck-like protein containing a CARD) or Nalp3. (a and b) Sw.71 trophoblast cells were transfected to express either small hairpin (sh) RNA shRNA for ASC (sh-ASC) or a control sequence (sh-control) (a) Western blot of lysates from the Sw.71 trophoblast cells expressing either sh-control or sh-ASC for ASC expression. $\beta$ actin served as a loading control. (b) Thirty-six hours after either non-infected (NI) or Chlamydia trachomatis (Ct) infection, levels of secreted IL-1 $\beta$ in the supernatants of sh-control and sh-ASC cells were measured by enzyme-linked immunosorbent assay (ELISA). $n=3$; ${ }^{*} P<0.05$ relative to the $\mathrm{NI}$ control. (c-e) Sw.71 trophoblast cells were transfected to express either shRNA for Nalp3 (sh-Nalp3) or a control sequence (sh-control). (c) RNA from the Sw.71 trophoblast cells expressing either sh-control or sh-NAlp3 was analyzed for Nalp3 mRNA expression by quantitative real-time reversetranscriptase-PCR. $n=3$; ${ }^{*} P<0.05$ relative to the sh-control. (d) Seventy-two hours after either no treatment (NT) or treatment with monosodium urate (MSU) $\left(100 \mu \mathrm{g} \mathrm{ml}^{-1}\right)$ levels of secreted IL-1 $\beta$ in the supernatants of sh-control and sh-Nalp3 cells were measured by ELISA. $n=3$; ${ }^{*} P<0.05$ relative to the NT control unless otherwise indicated. (e) Thirty-six hours after either NI or Ct infection, levels of secreted IL-1 $\beta$ in the supernatants of sh-control and sh-Nalp3 cells were measured by ELISA. $n=3 ;{ }^{\star} P<0.05$ relative to the $\mathrm{NI}$ control.

\section{DISCUSSION}

The trophoblast develops from the blastocyst to form the placenta. These cells provide an interface between the fetus and the mother and invade deep into the maternal endometrium (decidua) to remodel the maternal vasculature. ${ }^{39}$ They are distinctive cells that constitutively secrete chemokines, such as IL-8, monocyte chemoattractant protein-1, and growth-related oncogene $\alpha$, which are important for the normal recruitment of innate immune cells to the maternal-fetal interface, in the absence of a threat. ${ }^{40}$ Like cells of the innate immune system, 
a

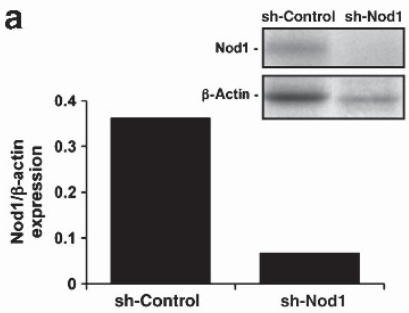

e

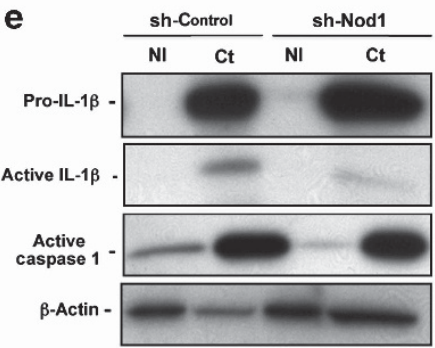

b
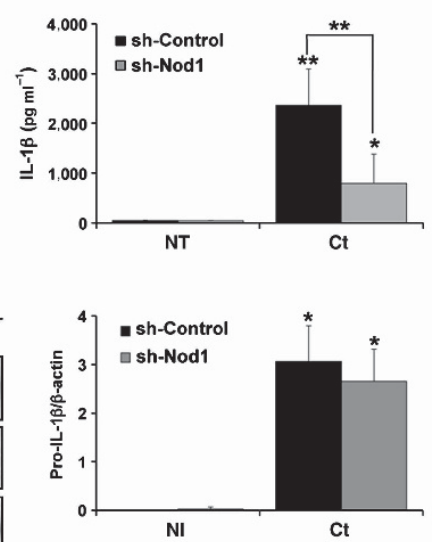
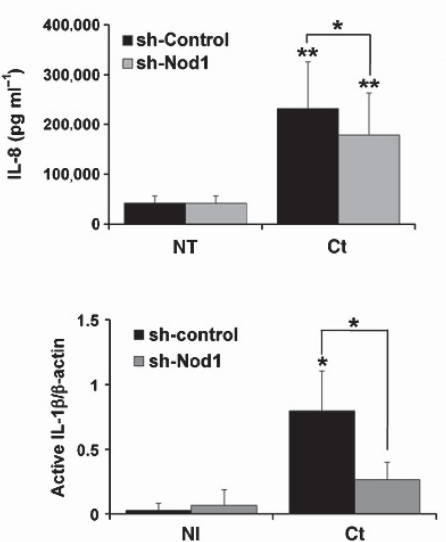

d
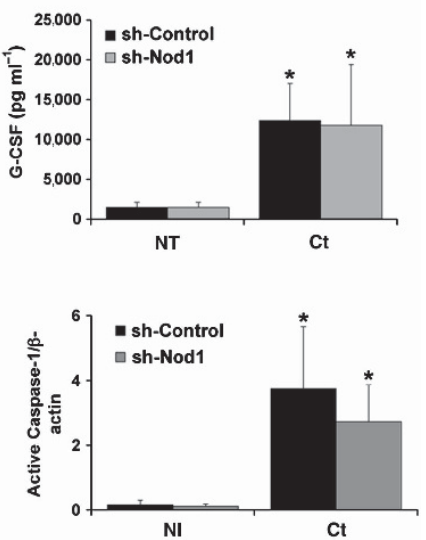

Figure 5 Chlamydia-induced trophoblast interleukin (IL)-1 $\beta$ processing and secretion is dependent on Nod1. Sw.71 trophoblast cells were transfected to express either small hairpin (sh) RNA for Nod1 (sh-Nod1) or a control sequence (sh-control) and were either non-infected (NI) or infected with Chlamydia trachomatis (Ct) for $36 \mathrm{~h}$. (a) Expression of Nod1 protein in the sh-control and sh-Nod1 cells was determined by western blot analysis, and bar chart shows densitometry with Nod1 expression normalized to $\beta$-actin levels. (b-d) Bar charts show levels of secreted IL-1 $\beta$, IL-8 and granulocyte colony-stimulating factor (G-CSF) in the supernatants of the sh-control and sh-Nod1 cells. (e) Supernatants were evaluated for proand active IL-1 $\beta$ and active caspase-1 expression by western blot. Bar charts show quantification of protein expression in the sh-control and sh-Nod1 cells as determined by densitometry and normalized to $\beta$-actin. $n=4 ;{ }^{*} P<0.05 ;{ }^{* \star} P<0.001$ relative to the $\mathrm{NI}$ control unless otherwise indicated.

the trophoblast express many functional PRRs and are able to sense a wide variety of pathogens and infectious components, leading to a range of responses from pro-inflammatory and anti-microbial/viral, to pro-apoptotic. ${ }^{15,16}$ We previously demonstrated that $\mathrm{Ct}$ infection of human first trimester trophoblast cells induces secretion of the potent pro-inflammatory cytokine, IL- $1 \beta .{ }^{11}$ In this current study, we report that Ct-induced trophoblast IL- $1 \beta$ secretion is associated with the transcription of IL- $1 \beta$ mRNA, the translation and processing of pro-IL- $1 \beta$, and the activation of caspase-1. In addition, we report, for the first time, the importance of Nod1 for mediating trophoblast IL- $1 \beta$ secretion in response to a Ct infection.

Using two human first trimester trophoblast cell lines (HTR8 and Sw.71), we found that Ct infection increases IL-1 $\beta$ mRNA expression and induces the expression of intracellular pro-IL1 $\beta$ protein. Interestingly, although non-infected trophoblast cells had little or no detectable levels of intracellular pro-IL-1 $\beta$, we were able to detect intracellular active caspase- 1 and active IL-1 $\beta$. However, Ct infection had no effect on the intracellular expression levels of active caspase- 1 and IL-1 $\beta$. By contrast, active IL-1 $\beta$ and active caspase- 1 were detected in trophoblast culture supernatants, but only following $\mathrm{Ct}$ infection. These findings suggest that there are intracellular stores of bioactive IL- $1 \beta$ in the trophoblast that are released soon after interaction with $\mathrm{Ct}$; and that IL- $1 \beta$ secretion in response to Ct infection is further increased over time following the strong induction of pro-IL-1 $\beta$ expression, active caspase- 1 , and IL- $1 \beta$ processing. These observations in the trophoblast are supported by studies in monocytes showing that these cells exhibit constitutive caspase-1 activation, leading to the rapid release of active IL- $1 \beta$ after a single signal, ${ }^{19}$ and that active caspase- 1 can be externalized. ${ }^{41}$
In order to elucidate the molecular mechanism for the production and secretion of IL- $1 \beta$ by the trophoblast after Ct infection of human trophoblast, we targeted PRRs known to be associated with cellular responses to chlamydia. ${ }^{20-31}$ Although TLRs are normally located on the plasma membrane, TLR2 and MyD88 can localize to the inclusion membrane of Ct-infected cells and mediate cytokine production. ${ }^{20}$ In a model of genital tract infection, TLR2 was shown to be important for Ct-induced cytokine production and oviduct pathology, without affecting the course of infection. By contrast, TLR4 knockout mice had normal pathology. ${ }^{22}$ MyD88, which is part of the signaling pathways of most TLRs and the IL-1 receptor, ${ }^{17}$ has also been shown to be important for Ct-induced cytokine production, including IL-1 $\beta .{ }^{21,29}$ However, in our system, using DN trophoblast cell lines, previously characterized, ${ }^{35-37}$ we found that the inhibition of TLR2, TLR4, or MyD88 function had no effect on the ability of Ct to induce intracellular pro-IL- $1 \beta$ expression or IL- $1 \beta$ secretion.

As the TLR/MyD88 pathway was not involved in Ct-induced IL- $1 \beta$ production by the trophoblast, we turned our attention to the role of the Nalp3/ASC inflammasome. In other cell types, Ct-induced IL- $1 \beta$ transcription, processing, and secretion have been shown to be mediated by the Nalp3/ASC inflammasome, ${ }^{29,31}$ and Ct-induced caspase- 1 activation and IL- $1 \beta$ processing is associated with pathology in vivo. ${ }^{42}$ Similarly, C. pneumoniae infection of macrophages induces IL- $1 \beta$ via the Nalp3 inflammasome, ${ }^{43,44}$ but in contrast to the Ct model, caspase-1-mediated IL-1 $\beta$ secretion is critical for resolution of the infection and protection against pathology. ${ }^{43,44}$ Nonetheless, these studies have demonstrated a role for Nalp3 and ASC in chlamydia-induced IL-1 $\beta$. By knocking down Nalp3 and ASC expression in the Sw.7 trophoblast cell line, we found that 
Ct-induced intracellular pro-IL- $1 \beta$ expression and IL- $1 \beta$ secretion were not dependent on either Nalp3 or ASC. This was further supported by our observation that the HTR8 cell line, which lacks ASC, ${ }^{34}$ also produces IL- $1 \beta$ in response to $\mathrm{Ct}$ infection. ${ }^{11}$ This lack of Nalp3 and ASC involvement in Ct-triggered IL-1 $\beta$ production by the Sw.71 cells is not due to the absence of inflammasome function in these cells. The Nalp3 agonist MSU was able to induce IL-1 $\beta$ secretion in Sw.71 cells, and this secretion was inhibited when Nalp3 and ASC expression were reduced. ${ }^{34}$

Another group of PRRs associated with chlamydia infection is the Nod proteins, Nod1 and Nod2, which recognize peptidoglycan peptides. ${ }^{16}$ Although peptidoglycan has not been detected biochemically in chlamydiae, both $\mathrm{Ct}$ and $C$. muridarum produce a rudimentary proteoglycan motif that is recognized by Nod1, leading to activation of nuclear factor $\kappa \mathrm{B}(\mathrm{NF} \kappa \mathrm{B})$ and pro-inflammatory cytokines. ${ }^{27,28}$ In addition, C. pneumoniae has been shown to induce an inflammatory response via Nod 1 and Nod $2 .{ }^{30}$ However, none of these studies reported whether the Nod proteins were involved in chlamydia-induced IL-1 $\beta$ production. As the HTR8 trophoblast cells produce IL-1 $\beta$ after Ct infection, but lack Nod $2,{ }^{38}$ we focused on the role of trophoblast Nod1 in Ct-induced IL-1 1 . Although knockdown of Nod1 expression in the Sw.71 trophoblast cells inhibited Ct-induced IL- $1 \beta$ secretion, we did not observe a decrease in either proIL-1 $\beta$ expression or caspase-1 activation. Because the Nod 1 protein has a CARD domain, its activation can lead to activation of caspase- 1 and IL- $1 \beta$ production. ${ }^{45}$ This dichotomy could have several explanations. Firstly, there are other NLRs that activate caspase-1 in an ASC-independent manner, such as Nlrc4 (Ipaf) or NLRs that have a CARD but not a pyrin domain. ${ }^{19}$ Thus, other NLRs, in addition to Nod1, may be triggered by Ct infection. Secondly, Ct-induced IL-1 $\beta$ processing in the trophoblast may be independent of caspase-1. Indeed, alternative mechanisms for Ct-induced IL-1 $\beta$ maturation include cleavage by serine proteases, typically produced by neutrophils and monocytes, ${ }^{46}$ and matrix metalloproteinases (MMPs), such as MMP-9 and MMP- $2,{ }^{47}$ which are highly expressed by the trophoblast. ${ }^{48}$

How Nod1 is triggering trophoblast secretion of IL- $1 \beta$ following $\mathrm{Ct}$ infection still remains to be determined. As IL- $1 \beta$ does not have a hydrophobic signal sequence required for entering the classical secretory pathway, hypothesized non-classical pathways of IL- $1 \beta$ release involve exocytosis of secretory lysosomes or exosomes, shedding of plasma membrane microvesicles, and export through the plasma membrane using specialized transporters. ${ }^{49}$ Trophoblast cells are known to constitutively release microvesicles and exosomes under normal physiological conditions, and this shedding from the placenta is elevated in pathological pregnancies. ${ }^{50}$ Moreover, these microparticles are known to contain bioactive proteins. ${ }^{50,51}$ In addition, Ct infection of THP- 1 macrophages (monocytic cell line) induced non-classical secretion of macrophage migration inhibitory factor by association with a Golgi complex protein $\mathrm{p} 115 .{ }^{52}$ Therefore, Nod1-induced IL-1 $\beta$ release by Ct infected trophoblast cells may be mediated by non-classical export pathways.

Another interesting finding to come out of these studies was that Ct-induced IL-8 secretion by the trophoblast was independent of TLR2 and TLR4, but dependent upon MyD88 and Nod1. One possible explanation for this finding is that induction of IL-8 may proceed through an IL-1 $\beta$ autocrine loop, as the IL-1 receptor signals through MyD88. ${ }^{17}$ This has been reported in other systems. In macrophages, the Nod2 agonist, muramyl dipeptide, induces IL-1 $\beta$ secretion, which in turn triggers the production of other cytokines, including IL- 8 via IL- $1 \beta .{ }^{53}$ Interestingly, although Nod1 mediated Ct-induced secretion of IL-1 $\beta$ and IL- 8 , Ct-induced G-CSF secretion occurred independently of Nod1, again highlighting that there may be multiple immune pathways activated upon Ct infection.

There is a well-established link between bacterial infections and adverse pregnancy outcomes, such as preterm birth, ${ }^{54}$ and IL- $1 \beta$ is an important mediator of preterm labor. ${ }^{55}$ Therefore, understanding the innate immune pathways activated by pathogens that lead to IL- $1 \beta$ production is important. Ct and C. pneumoniae have been detected in the decidual and placental tissues from complicated pregnancies ${ }^{6-9}$ and in mice, Ct infection triggers preterm delivery. ${ }^{56}$ Therefore, the ability of chlamydia to induce IL- $1 \beta$ at the maternal-fetal interface could have a negative impact on pregnancy outcome and a woman's reproductive potential. Our work establishes that $\mathrm{Ct}$ infection of the trophoblast activates the Nod1 pathway, leading to IL-1 $\beta$ secretion. As functional Nod1 is expressed by the trophoblast across gestation, ${ }^{57}$ it can be a target for pathogens throughout pregnancy. Indeed, administration of the Nod 1 agonist, iEDAP, to pregnant mice induces preterm delivery, reduces fetal weight, and induces an inflammatory response. ${ }^{57}$ Thus, targeting the Nod1 pathway may be useful to diminish preterm labor associated with bacterial infections.

\section{METHODS}

Reagents and antibodies. MSU was purchased from InvivoGen (San Diego, CA). Rabbit polyclonal antibody to IL-1 $\beta$ (\#2022), which recognizes the full-length pro and the processed active forms, was purchased from Cell Signaling (Danvers, MA). Rabbit polyclonal antibody to active caspase-1 (\#06-503) was purchased from Upstate (Lake Placid, NY). The rabbit anti-ASC polyclonal antibody was obtained from Calbiochem (Gibbstown, NJ). The rabbit polyclonal antibody recognizing Nod1 was purchased from (Imgenex, San Diego, $\mathrm{CA})$. The rabbit polyclonal antibody for human $\beta$-actin was purchased from Sigma (St Louis, MO).

Trophoblast cell lines. Three human first trimester trophoblast cell lines were used in these studies: the SVneo-transformed HTR8 cells; 58 the SVneo-transformed 3A cells; ${ }^{37}$ and telomerase-transformed Sw.71 cells. ${ }^{59}$ HTR8 and 3 A cells were cultured in RPMI and Sw.71 cells were cultured in Dulbecco's modified Eagle's medium (Gibco-Invitrogen; Grand Island, NY). Both media were supplemented with 10\% fetal bovine serum (Hyclone, South Logan, UT), 10 mm Hepes (hydroxyethyl piperazineethanesulfonic acid), $0.1 \mathrm{~mm}$ modified Eagle's medium non-essential amino acids, $1 \mathrm{~mm}$ sodium pyruvate, and $100 \mathrm{~nm}$ penicillin/streptomycin (Gibco-Invitrogen). Cells were maintained at $37^{\circ} \mathrm{C} / 5 \% \mathrm{CO}_{2}$. In previous studies, we have shown that the HTR8 cells constitutively lack Nod2 and ASC expression..$^{34,38}$

Stably transfected trophoblast cell lines. HTR8 cells were stably transfected with either the pDeNy plasmid containing the human MyD88 DN (MyD88-DN), or the pZERO plasmid containing the human TLR4$\mathrm{DN}$ (Invivogen), as previously described. ${ }^{35,36}$ The $3 \mathrm{~A}$ cells were stably 
transfected with the pZERO plasmid containing the human TLR2-DN (Invivogen) as previously described. ${ }^{37}$ The Sw.71 cells were stably transfected with the pLKO.1 expression plasmids containing the ASC-shRNA construct, NM_013258.3-718s1c1 (sh-ASC), or a non-target shRNA control, SHC002 (sh-control) from Sigma Aldrich (St Louis, MO), as previously described. ${ }^{34}$ The Sw.71 cells were also transfected with specific shRNA Nalp3-shRNA (sh-Nalp3) or a mutated targeting sequence as a negative control (sh-mut), which were both a kind gift from Dr Jenny Ting (Lineberger Comprehensive Cancer Center, Chapel Hill, NC) ${ }^{60}$ Knockdown of Nod1 (sh-Nod1) was performed using a specific shRNA targeting sequence, NOD1 NM_006092.1 from Sigma.

Infection of trophoblast cells with Ct. Ct serovar D (rifampin-resistant) was a gift from Dr. Robert DeMars (University of Wisconsin, Madison, WI). Ct was propagated in HeLa cells grown in antibiotic-free Dulbecco's modified Eagle's medium (Gibco-Invitrogen) and stock cultures generated as previously described. ${ }^{11}$ Trophoblast cells $\left(1 \times 10^{5}\right)$ were seeded into wells of a six-well plate and allowed to attach overnight. The next day, the cells were washed with $10 \mathrm{ml}$ of phosphate-buffered saline (PBS) and infected at a multiplicity of infection of 1 in $2 \mathrm{ml}$ of sucrose phosphate glutamate by spinning at $350 \times g$ at $8-10^{\circ} \mathrm{C}$ for $40 \mathrm{~min}$ in an Eppendorf centrifuge with a plate holder. This multiplicity of infection was calculated based on an assay performed using HeLa cells. ${ }^{11}$ The trophoblast cells were then washed with PBS to remove any unattached bacteria. Fresh serum-free OptiMEM (Gibco-Invitrogen) was then added to the plates, and the cells were cultured at $37^{\circ} \mathrm{C}$ for $12-48 \mathrm{~h}$, after which cell-free supernatants were collected and cells lysed for either RNA or protein isolation. Infection rates were determined by staining the cells with an anti-lipopolysaccharide antibody to $\mathrm{Ct}$, and analysis performed by flow cytometry as previously described. ${ }^{11}$

Quantitative real-time reverse-transcriptase-PCR. Total RNA was isolated from cells using the RNeasy Mini Prep kit from Qiagen (Valencia, CA) following the manufacturer's protocol. Reverse transcription of the RNA into cDNA was done on $5 \mu \mathrm{g}$ of total RNA using the Sprint RT Complete Oligo (dT) 18 kit from Clontech (Mountain View, CA) following the manufacturer's protocol. Quantitative real-time reversetranscriptase-PCR was performed using commercially available Applied Biosystems (Carlsbad, CA) probes for IL-1 $\beta$ (Hs00174097_m1), Nalp3 (Hs00918082_m1), and the control GAPDH (glyceraldehyde-3-phosphate dehydrogenase; Hs99999905_m1), Taqman Universal Mastermix, and a Thermal Cycler (iQ5; Bio-Rad Laboratories, Hercules, CA). Samples were analyzed in duplicate and the target gene expression was normalized to GAPDH.

Western blot analysis. For analysis of proteins by western blot, proteins diluted to $20 \mu \mathrm{g}$ with gel-loading buffer were boiled for $5 \mathrm{~min}$ and resolved under reducing conditions on 12\% SDS-PAGE (sodium dodecyl sulfate-polyacrylamide gel electrophoresis) gels and then transferred onto polyvinylidene difluoride paper (PerkinElmer, Boston, MA). Membranes were blocked with 5\% fat-free powdered milk in PBS/0.05\% Tween-20 (PBS-T). Following washes with PBS-T, membranes were incubated overnight at $4{ }^{\circ} \mathrm{C}$ with primary antibody in $\mathrm{PBS}-\mathrm{T} / 1 \%$ fat-free powdered milk. Following this incubation, membranes were washed as before and then incubated with the goat anti-rabbit IgG secondary antibody conjugated to peroxidase (Vector Labs; Burlingame, CA) in PBS-T/1\% fat-free powdered milk. Following washes with PBS-T and then with distilled water, the peroxidase-conjugated antibody was detected by enhanced chemiluminescence (PerkinElmer). $\beta$-actin was used as internal control, in addition to Ponseau Red, to validate the amount of protein loaded onto the gels. Images were recorded and semi-quantitative densitometry performed using the Gel Logic 100 and Kodak MI software (Eastman Kodak, Rochester, NY).

Cytokine studies. Trophoblast culture supernatants were analyzed for IL- $1 \beta$ and IL-8 using commercial enzyme-linked immunosorbent assay kits from R\&D Systems (Minneapolis, MN) and Enzo Life
Sciences (Farmingdale, NY), respectively. IL-1 $\beta$, IL-8, and G-CSF were also measured using the Human BioPLex assay (BioRad, Hercules, CA) with detection and analysis performed using the Luminex 100 IS system (Millipore, Billerica, MA).

Statistical analysis. Data are expressed as mean \pm s.d. All experiments were performed at least three times and post-analysis data were pooled. Statistical significance $(P<0.05)$ was determined using either the Student's $t$-tests or, for multiple comparisons, one-way analysis of variance followed by Bonferroni's post-test.

\section{ACKNOWLEDGMENTS}

This work was supported by NIH/NIAID grants R01AI049571 (to PBK) and $\mathrm{NIH/NICHD}$ grants RO1HD049446 (to VMA) and PO1HD054713 (to VMA).

\section{NOTE ADDED IN PROOF}

After review of this manuscript, the authors found a relevant paper that also supports their findings for a lack of a role of the ASC inflammasome in IL-1 $\beta$ responses to Chlamydia. In this study, Nagarajan et al., reported that Chlamydia induced IL-1 and oviduct pathology using an in vivo model, but that the ASC inflammasome was not involved [Nagarajan UM, Sikes JD, Yeruva L, Prantner D. Significant role of IL-1 signaling, but limited role of inflammasome activation, in oviduct pathology during Chlamydia muridarum genital infection. J Immunol. 188, 2866-2875 (2012)].

\section{DISCLOSURE}

The authors declared no conflict of interest.

\section{(C) 2013 Society for Mucosal Immunology}

\section{REFERENCES}

1. Mylonas, I. Female genital Chlamydia trachomatis infection: where are we heading?. Arch. Gynecol. Obstet. 285, 1271-1285 (2012).

2. Wiesenfeld, H.C. et al. Lower genital tract infection and endometritis: insight into subclinical pelvic inflammatory disease. Obstet. Gynecol. 100, 456-463 (2002).

3. Baud, D., Regan, L. \& Greub, G. Emerging role of Chlamydia and Chlamydia-like organisms in adverse pregnancy outcomes. Curr. Opin. Infect. Dis. 21, 70-76 (2008).

4. Howie, S.E., Horner, P.J. \& Horne, A.W. Chlamydia trachomatis infection during pregnancy: known unknowns. Discov. Med. 12, 57-64 (2011).

5. Dong, Z.W., Li, Y., Zhang, L.Y. \& Liu, R.M. Detection of Chlamydia trachomatis intrauterine infection using polymerase chain reaction on chorionic villi. Int. J. Gynaecol. Obstet. 61, 29-32 (1998).

6. Magon, T., Kluz, S., Chrusciel, A., Obrzut, B. \& Skret, A. The PCR assessed prevalence of Chlamydia trachomatis in aborted tissues. Med. Wieku. Rozwoj. 9, 43-48 (2005).

7. Gomez, L.M. \& Parry, S. Trophoblast infection with Chlamydia pneumoniae and adverse pregnancy outcomes associated with placental dysfunction. Am. J. Obstet. Gynecol. 200, 526 e521-527 (2009).

8. Baud, D. et al. Role of Chlamydia trachomatis in miscarriage. Emerg. Infect. Dis. 17, 1630-1635 (2011).

9. Rours, G.I. et al. Chlamydia trachomatis and placental inflammation in early preterm delivery. Eur. J. Epidemiol. 26, 421-428 (2011).

10. Azenabor, A.A., Kennedy, P. \& Balistreri, S. Chlamydia trachomatis infection of human trophoblast alters estrogen and progesterone biosynthesis: an insight into role of infection in pregnancy sequelae. Int. J. Med. Sci. 4, 223-231 (2007).

11. de la Torre, E., Mulla, M.J., Yu, A.G., Lee, S.J., Kavathas, P.B. \& Abrahams, V.M. Chlamydia trachomatis infection modulates trophoblast cytokine/chemokine production. J. Immunol. 182, 3735-3745 (2009).

12. Salaun, B., Romero, P. \& Lebecque, S. Toll-like receptors' two-edged sword: when immunity meets apoptosis. Eur. J. Immunol. 37, 3311-3318 (2007).

13. Bortoluci, K.R. \& Medzhitov, R. Control of infection by pyroptosis and autophagy: role of TLR and NLR. Cell Mol. Life Sci. 67, 1643-1651 (2010).

14. Kumar, H., Kawai, T. \& Akira, S. Pathogen recognition by the innate immune system. Int. Rev. Immunol. 30, 16-34 (2011). 
15. Abrahams, V.M. Pattern recognition at the maternal-fetal interface. Immunol. Invest. 37, 427-447 (2008).

16. Abrahams, V.M. The role of the Nod-like receptor family in trophoblast innate immune responses. J. Reprod. Immunol. 88, 112-117 (2011).

17. Brikos, C. \& O'Neill, L.A. Signalling of toll-like receptors. Handb. Exp. Pharmacol. 183), 21-50 (2008).

18. Philpott, D.J. \& Girardin, S.E. Nod-like receptors: sentinels at host membranes. Curr. Opin. Immunol. 22, 428-434 (2010).

19. Netea, M.G., Simon, A., van de Veerdonk, F., Kullberg, B.J., Van der Meer, J.W. \& Joosten, L.A. IL-1beta processing in host defense: beyond the inflammasomes. PLoS Pathog. 6, e1000661 (2010).

20. O'Connell, C.M., Ionova, I.A., Quayle, A.J., Visintin, A. \& Ingalls, R.R. Localization of TLR2 and MyD88 to Chlamydia trachomatis inclusions. Evidence for signaling by intracellular TLR2 during infection with an obligate intracellular pathogen. J. Biol. Chem. 281, 1652-1659 (2006).

21. Nagarajan, U.M., Ojcius, D.M., Stahl, L., Rank, R.G. \& Darville, T. Chlamydia trachomatis induces expression of IFN-gamma-inducible protein 10 and IFN-beta independent of TLR2 and TLR4, but largely dependent on MyD88. J. Immunol. 175, 450-460 (2005).

22. Darville, T., O'Neill, J.M., Andrews, C.W. Jr, Nagarajan, U.M., Stahl, L. \& Ojcius, D.M. Toll-like receptor-2, but not Toll-like receptor-4, is essential for development of oviduct pathology in chlamydial genital tract infection. J. Immunol. 171, 6187-6197 (2003).

23. Bas, S. et al. The proinflammatory cytokine response to Chlamydia trachomatis elementary bodies in human macrophages is partly mediated by a lipoprotein, the macrophage infectivity potentiator, through TLR2/TLR1/TLR6 and CD14. J. Immunol. 180, 1158-1168 (2008).

24. Shaw, J.L. et al. Chlamydia trachomatis infection increases fallopian tube PROKR2 via TLR2 and NFkappaB activation resulting in a microenvironment predisposed to ectopic pregnancy. Am. J. Pathol. 178, 253-260 (2011).

25. Mackern-Oberti, J.P., Maccioni, M., Cuffini, C., Gatti, G. \& Rivero, V.E. Susceptibility of prostate epithelial cells to Chlamydia muridarum infection and their role in innate immunity by recruitment of intracellular Toll-like receptors 4 and 2 and MyD88 to the inclusion. Infect. Immun. 74, 6973-6981 (2006).

26. Romano Carratelli, C., Mazzola, N., Paolillo, R., Sorrentino, S. \& Rizzo, A. Toll-like receptor-4 (TLR4) mediates human beta-defensin-2 (HBD-2) induction in response to Chlamydia pneumoniae in mononuclear cells. FEMS Immunol. Med. Microbiol. 57, 116-124 (2009).

27. Buchholz, K.R. \& Stephens, R.S. The cytosolic pattern recognition receptor NOD1 induces inflammatory interleukin-8 during Chlamydia trachomatis infection. Infect. Immun. 76, 3150-3155 (2008).

28. Welter-Stahl, L. et al. Stimulation of the cytosolic receptor for peptidoglycan, Nod1, by infection with Chlamydia trachomatis or Chlamydia muridarum. Cell Microbiol. 8, 1047-1057 (2006).

29. Abdul-Sater, A.A., Said-Sadier, N., Padilla, E.V. \& Ojcius, D.M. Chlamydial infection of monocytes stimulates IL-1 beta secretion through activation of the NLRP3 inflammasome. Microbes Infect. 12, 652-661 (2010).

30. Opitz, B. et al. Nod1-mediated endothelial cell activation by Chlamydophila pneumoniae. Circ. Res. 96, 319-326 (2005).

31. Abdul-Sater, A.A., Koo, E., Hacker, G. \& Ojcius, D.M. Inflammasomedependent caspase-1 activation in cervical epithelial cells stimulates growth of the intracellular pathogen Chlamydia trachomatis. J. Biol. Chem. 284, 26789-26796 (2009).

32. van Westreenen, M., Pronk, A., Diepersloot, R.J., de Groot, P.G. \& Leguit, P. Chlamydia trachomatis infection of human mesothelial cells alters proinflammatory, procoagulant, and fibrinolytic responses. Infect. Immun. 66, 2352-2355 (1998).

33. Gervassi, A., Alderson, M.R., Suchland, R., Maisonneuve, J.F., Grabstein, K.H. \& Probst, P. Differential regulation of inflammatory cytokine secretion by human dendritic cells upon Chlamydia trachomatis infection. Infect. Immun. 72, 7231-7239 (2004).

34. Mulla, M.J. et al. Uric acid induces trophoblast IL-1beta production via the inflammasome: implications for the pathogenesis of preeclampsia. Am. J. Reprod. Immunol. 65, 542-548 (2011).

35. Carroll, T.Y. et al. Modulation of trophoblast angiogenic factor secretion by antiphospholipid antibodies is not reversed by heparin. Am. J. Reprod. Immunol. 66, 286-296 (2011).

36. Mulla, M.J. et al. Antiphospholipid antibodies induce a pro-inflammatory response in first trimester trophoblast via the TLR4/MyD88 pathway. Am. J. Reprod. Immunol. 62, 96-111 (2009).
37. Abrahams, V.M. et al. TLR6 modulates first trimester trophoblast responses to peptidoglycan. J. Immunol. 180, 6035-6043 (2008).

38. Costello, M.J., Joyce, S.K. \& Abrahams, V.M. NOD protein expression and function in first trimester trophoblast cells. Am. J. Reprod. Immunol. 57, 67-80 (2007).

39. Cartwright, J.E., Fraser, R., Leslie, K., Wallace, A.E. \& James, J.L. Remodelling at the maternal-fetal interface: relevance to human pregnancy disorders. Reproduction 140, 803-813 (2010).

40. Mor, G., Romero, R., Aldo, P.B. \& Abrahams, V.M. Is the trophoblast an immune regulator? The role of Toll-like receptors during pregnancy. Crit. Rev. Immunol. 25, 375-388 (2005).

41. Laliberte, R.E., Eggler, J. \& Gabel, C.A. ATP treatment of human monocytes promotes caspase-1 maturation and externalization. J. Biol. Chem. 274, 36944-36951 (1999).

42. Cheng, W., Shivshankar, P., Li, Z., Chen, L., Yeh, I.T. \& Zhong, G. Caspase-1 contributes to Chlamydia trachomatis-induced upper urogenital tract inflammatory pathologies without affecting the course of infection. Infect. Immun. 76, 515-522 (2008).

43. Shimada, K. et al. Caspase-1 dependent IL-1 beta secretion is critical for host defense in a mouse model of Chlamydia pneumoniae lung infection. PLoS One 6, e21477 (2011).

44. He, X., Mekasha, S., Mavrogiorgos, N., Fitzgerald, K.A., Lien, E. \& Ingalls, R.R. Inflammation and fibrosis during Chlamydia pneumoniae infection is regulated by IL-1 and the NLRP3/ASC inflammasome. J. Immunol. 184, 5743-5754 (2010).

45. Yoo, N.J. et al. Nod1, a CARD protein, enhances pro-interleukin-1beta processing through the interaction with pro-caspase-1. Biochem. Biophys. Res. Commun. 299, 652-658 (2002).

46. Prantner, D. et al. Critical role for interleukin-1 beta (IL-1beta) during Chlamydia muridarum genital infection and bacterial replicationindependent secretion of IL-1beta in mouse macrophages. Infect. Immun. 77, 5334-5346 (2009).

47. Russo, R. et al. Evidence implicating matrix metalloproteinases in the mechanism underlying accumulation of IL-1beta and neuronal apoptosis in the neocortex of HIV/gp120-exposed rats. Int. Rev. Neurobiol. 82, 407-421 (2007).

48. Ferretti, C., Bruni, L., Dangles-Marie, V., Pecking, A.P. \& Bellet, D. Molecular circuits shared by placental and cancer cells, and their implications in the proliferative, invasive and migratory capacities of trophoblasts. Hum. Reprod. Update 13, 121-141 (2007).

49. Eder, C. Mechanisms of interleukin-1 beta release. Immunobiology 214, 543-553 (2009).

50. Holder, B.S., Tower, C.L., Forbes, K., Mulla, M.J., Aplin, J.D. \& Abrahams, V.M. Immune cell activation by trophoblast-derived microvesicles is mediated by syncytin 1. Immunology 136, 184-191 (2012).

51. Mincheva-Nilsson, L. \& Baranov, V. The role of placental exosomes in reproduction. Am. J. Reprod. Immunol. 63, 520-533 (2010).

52. Merk, M. et al. The Golgi-associated protein $\mathrm{p} 115$ mediates the secretion of macrophage migration inhibitory factor. J. Immunol. 182, 6896-6906 (2009).

53. Hedl, M. \& Abraham, C. Distinct roles for Nod2 protein and autocrine interleukin-1 beta in muramyl dipeptide-induced mitogen-activated protein kinase activation and cytokine secretion in human macrophages. J. Biol. Chem. 286, 26440-26449 (2011).

54. Lamont, R.F. The role of infection in preterm labour and birth. Hosp. Med. 64, 644-647 (2003).

55. Kemp, M.W., Saito, M., Newnham, J.P., Nitsos, I., Okamura, K. \& Kallapur, S.G. Preterm birth, infection, and inflammation advances from the study of animal models. Reprod. Sci. 17, 619-628 (2010).

56. Pal, S., Peterson, E.M. \& De La Maza, L.M. A murine model for the study of Chlamydia trachomatis genital infections during pregnancy. Infect. Immun. 67, 2607-2610 (1999).

57. Cardenas, I. et al. Nod1 activation by bacterial iE-DAP induces maternalfetal inflammation and preterm labor. J. Immunol. 187, 980-986 (2011).

58. Graham, C.H. et al. Establishment and characterization of first trimester human trophoblast cells with extended lifespan. Exp. Cell Res. 206, 204-211 (1993).

59. Straszewski-Chavez, S.L. et al. Isolation and characterization of a novel telomerase immortalized first trimester trophoblast cell line, Swan 71. Placenta 30, 939-948 (2009).

60. Allen, I.C. et al. The NLRP3 inflammasome mediates in vivo innate immunity to influenza A virus through recognition of viral RNA. Immunity 30, 556-565 (2009). 